\title{
Fuel cells as key components for the next generation of autonomous diagnostic devices
}

\author{
N.Sabate ${ }^{1,2}$ \\ ${ }^{1}$ Instituto de Microelectrónica de Barcelona IMB-CNM (CSIC), C/ del Til·lers sn, Campus UAB, 08193 \\ Bellaterra, Barcelona, Spain \\ ${ }^{2}$ Catalan Institution for Research and Advanced Studies (ICREA), Passeig Lluís Companys 23, 08010 \\ Barcelona, Spain \\ neus.sabate@imb-cnm.csic.es
}

\begin{abstract}
We present a new generation of microfuel cells that take advantage of the enhanced properties of paper microfluidic platforms. Ultimately, these fuel cells are to be totally printed and compatible with the fabrication processes of current lateral flow tests so they can be readily integrated within the device to be powered. This will achieve simplicity, ease of use, portability and low environmental impact not offered by any of the state-of-the-art solutions.
\end{abstract}

Key words: fuel cells, paper diagnostic devices, autonomous devices, self-powered

\section{Introduction}

Since Whitesides and co-workers [1] introduced the idea of fabricating microfluidic channels on paper ( $\mu$ PADs) for multiplex analyte colorimetric detection in 2007, many new areas of exploration have opened up. Many exciting examples of paper-cut microfluidic devices that take advantage of filtration, reactant dilution, flow velocity control or sequential mixing driven by capillarity have been reported since then [2]. These new research avenues have resulted in paper-based sensors beyond colorimetric approaches, such as electrochemical, chemiluminescence or electrochemiluminescence $[3,4]$.

The particular features of this new generation of enhanced-performing rapid tests make them currently the most promising candidates to fulfill the guidelines set by the World Health Organization (WHO) for the development of diagnostics in resource-poor settings. These tests must be ASSURED (i.e. affordable, sensitive, specific, user-friendly, rapid and robust, equipment-free and delivered to those who need it) and according to the Millennium Development Goals agenda, they will enable local communities to improve healthcare, environmental safety and food quality.

The advances in paper microfluidics described so far show that the new generation of paperbased devices is able to overcome the limitations of traditional lateral flow tests because they will provide more accurate and specific information. Effective, quantitative and multiplexed tests can already be performed as far as an appropriate handheld or small benchtop reader is used. Indeed, signal quantification requires the use of instrumentation and today there are a number of commercial readers that can read colorimetric, fluorescent or electrochemical assays [5]. However, these readers are only cost-effective when used in hospitals or consultation rooms and eventually, to perform home-test monitoring of chronic diseases (e.g. glucometer) but cannot be dedicated to perform a single disposable test. This severely limits the applicability of paper-based sensors to close-topatient point-of-care. In the search of simpler approaches, the high market penetration of smartphones has opened a low-cost route to quantification of colorimetric paper-based assays through built-in cameras.

A more promising alternative are the on-chip approaches. It is surprising that despite the huge expected impact behind ASSURED assays, the search for effective on-chip solutions is still in its infancy. According to our opinion, the successful solutions will be the ones that offer on-chip readout capabilities without modifying significantly current lateral flow manufacturing processes. A key aspect to accomplish this vision is sufficient and available 
electrical power on-board. Ideally, the power source has to be affordable, paper-based and environmentally friendly when disposed of. In this sense, research in printed batteries - also in paper substrates - has been very active in the recent years.

\section{Paper-based biofuel cells}

Our research team is devoted to develop a new generation of disposable and low-environmental impact fuel cells [6]. In this approach, these devices are conceived to be single-use and disposable power sources. We think that this can have an enormous impact in the point-ofcare diagnostics domain, as it will provide simple, reliable and clean power sources to an upcoming generation of smart paper-based sensors that will allow them to be energetically autonomous. Ultimately, these fuel cells are to be manufactured in the same fabrication process and readily integrated within the lateral strip to be powered. Figure 1 represents the basic structure of one of these fuel cells. A biological fluid is added into the reception zone, and then flows by capillarity to a pre-treatment region, where, if required, it will be filtered or mixed with other reagents (mainly electrolytes). Once conditioned, the fluid will flow through the reaction zone where part of the electrochemical energy of the fluid will be converted into electrical power. Finally, the sample will reach the absorbent pad placed at the end of the paper strip. Fuel cell operating time will be determined by the flow rate established in the paper channel and the volumetric capacity of the absorbent pad. After that time lapse, the fuel cell can be disposed of together with the paper-based sensing module. In fact, the materials used in these devices (paper, carbonbased inks and ultra-low contents of metallic nanoparticles or enzymes) make them more environmentally friendly than batteries.

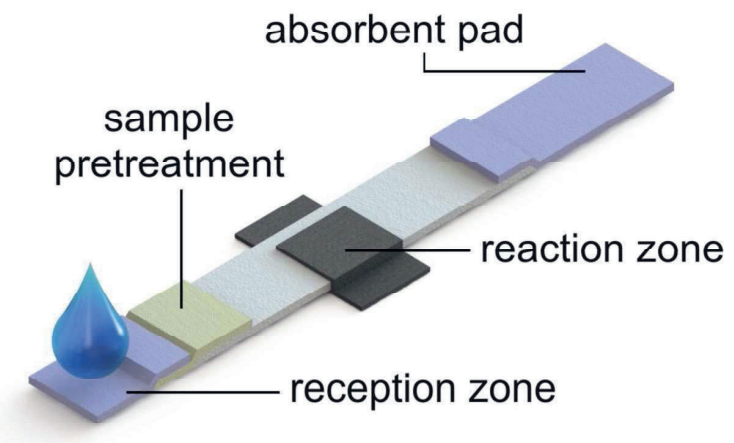

Fig. 1. Basic scheme of a paper-based microfluidic fuel cell

\section{Glucose fuel cells for energy generation energy with blood samples}

Paper glucose fuel cells are promising candidates to provide energy on-board to smart paper microfluidic devices. Ideally, the energy would be harvested from the blood sample to be analyzed. Blood is very relevant clinically as it provides direct information about patient health status. The range of application is huge: from relevant parameters of routine biochemical analysis (beyond glucose or cholesterol) to cancer markers or cardiovascular indicators. However, due to the limited power generated by these devices - in the range of hundreds of microwatts per $\mathrm{cm} 2$ at most $[7,8]$ - real applications are still scarce. Among the different hurdles that prevent these power sources to become effective components of the next generation of point-of-care devices, we can stress out the need of generating a useful voltage to power an electronic module, the capability of sustaining a constant current under resistive loads or the need of optimizing the faradic efficiency obtained from a small blood sample. The talk will address the state-of-theart and will point out the different strategies that can be employed to turn current prototypes into suitable power sources for the next generation of point-of-care devices.

\section{References}

[1] A.W. Martinez, et al., Lab Chip 8, 2146-2150 (2008)

[2] R.F.Carvalhal et al., Bioanalysis 7, 16631665 (2010)

[3] D.D. Liana et al., Sensors 12, 11505-11526 (2012)

[4] A.K.Yetisen et al., Lab Chip 13, 2210-2251 (2013)

[5] J.Hu et al., Biosensors and Bioelectronics 54, 585-597 (2014)

[6] J.P. Esquivel et al. Energy \& Environmental Science 7,1744-1749 (2014);

[7] M.J. Gonzalez-Guerrero et al. Biosensors and Bioelectronics 15, 475-480 (2017)

[8] I.Shitanda et al. Journal of Power Sources 360, 516-519 (2017) 